\title{
A novel intestinal organoid-based in vitro co-culture system to dissect out the initial host defense system
}

\author{
Atsushi Mizoguchi ${ }^{1}$
}

Received: 13 July 2016/Accepted: 13 July 2016/Published online: 23 July 2016

(C) Japanese Society of Gastroenterology 2016

The surface of intestine in adult humans, which spans an area of approximately $200-400 \mathrm{~m}^{2}$ as big as the area of tennis court [1], provides the first physiological defense against intestinal luminal contents, including a huge variety of microorganisms and food antigens [2]. The intraepithelial lymphocytes (IELs), which reside within the epithelium, contribute to restoring the integrity of the epithelial barrier to maintain the initial host defense against enteric microorganisms. In contrast, IELs can also damage epithelial cells and induce inflammatory responses through their cytotoxic activity and production of inflammatory cytokines such as IFN- $\gamma[3,4]$. IELs are characterized by extremely heterogeneous $\mathrm{T}$ cell populations, which are classified into "induced IELs", which develop through thymic selection and increase with age, and "natural IELs", which contain at least two unique T cell subsets that recognize self-antigens and non-peptide antigens [3, 4]. One subset in natural IELs expresses CD8 $\alpha \alpha$ homodimers and develops through "alternative positive selection". Another subset, which expresses T cell receptor (TCR) $\gamma \delta$, develops extrathymically, presents at birth, and decreases with age. However, the origin, development pathways, and functions of natural IELs have been under long-standing debates [3, 4].

To fully understand the complicated differentiation pathway of IELs and their functional networks with epithelial cells, in vitro co-culture systems of IELs with cancer cell lines have been established [5]. However, the physiological relevance of using cancer cell lines is

Atsushi Mizoguchi

mizoguchi_atsushi@med.kurume-u.ac.jp

1 Department of Immunology, Kurume University School of Medicine, Kurume, Japan questionable due to their already altered-function through the carcinogenetic process [6]. Alternatively, the nature of non-transformed epithelial cells that rapidly enter into programmed cell death, a phenomenon known as "anoikis" [7], has hampered investigators to utilize the freshly isolated intestinal epithelial cells in a physiological setting for a long-term co-culture with IELs. In this issue of Journal of Gastroenterology, Nozaki et al. introduce an organoid-based in vitro co-culture system with murine IELs to overcome the fundamental problems [8]. This novel co-culture system employs three-dimensional organoids in Matrigel composed of stem cell-derived crypt-villus structures without mesenchymal niche [9]. One of the advantages with this system is the prevention of anoikis. Importantly, there are different types of epithelial cells such as Paneth cells and Goblet cells in a crypt, which play distinct functions through the production of different factors such as cytokines. Therefore, the co-existence of different types of epithelial cells is necessary to allow an in vitro system to more closely reflect in vivo phenomena played by epithelial cells. In this regard, the organoid-based co-culture system that contains stem cell-derived different types of epithelial cells has a great advantage. Indeed, the advantage has allowed the authors to clearly demonstrate that small intestinal epithelial cells from mice can sufficiently support the expansion and survival of $\mathrm{TCR} \gamma \delta^{+}$IELs as well as TCR $\alpha \beta^{+}$IELs in both contact-dependent and -independent manners [8].

By employing a single-plane time-lapse imaging system, the authors then reveal that IELs are highly motile. IELs are found to migrate into, and then come out from, the organoids [8]. This finding is certainly of interest because it challenges current concepts that TCR $\gamma \delta^{+}$IELs do not recirculate and that intestinal epithelial space provides the 
final destination of $\mathrm{T}$ cells $[3,4]$. In addition, the authors demonstrate another novel finding that, during the movement, IELs can continuously change their cellular shapes [8]. As compared to the in vivo system, the organoid-based in vitro co-culture system therefore may provide a more useful tool to closely examine the interaction of epithelial cells and IELs at a cellular level. Small intestine contains at least ten times more IELs than the large intestine, which is continuously exposed to many more microorganisms [10]. One can therefore predict that some local factors such as epithelial cells, which exhibit functional and physiological differences between small and large intestines, may contribute for the determination of IEL development and expansion, but this issue still remains to be resolved. In addition, a recent report proposes that a subset of IELs may originate from Foxp $3^{+}$regulatory $\mathrm{T}$ cells (Treg) [11]. The organoid-based in vitro co-culture system in which organoids can be generated from small versus large intestinal epithelial cells and variety of $\mathrm{T}$ cell subsets can be used would be a useful tool to study the scientific topics in IEL biology.

It is becoming increasingly apparent that enteric microorganisms are involved in the pathogenesis of wide variety of inflammatory conditions ranging from local immune-mediated diseases such as inflammatory bowel disease to neurological disorders such as autism [12, 13]. The initial defense system maintained by the co-operation of IELs and epithelial cells plays a significant role in the regulation of host/microbial interactions, and IELs have been proposed to positively or negatively control the pathogenesis of not only intestinal disorders but also neurological disorders such as autoimmune encephalomyelitis $[14,15]$. Therefore, further extension of the organoid-based in vitro co-culture system in mice to humans would be a significant undertaking to provide an important rationale for developing novel preventive and therapeutic strategies for these disorders.

\section{Compliance with ethical standards}

Conflict of interest The authors declare that they have no conflict of interest.

\section{References}

1. Hooper LV, MacPherson AJ. Immune adaptations that maintain homeostasis with the intestinal microbiota. Nat Rev Immunol. 2010;10:159-69.

2. Okamoto R, Watanabe M. Role of epithelial cells in the pathogenesis and treatment of inflammatory bowel disease. J Gastroenterol. 2016;51:11-21.

3. Ishikawa $H$, Naito $T$, Iwanaga $T$, et al. Curriculum vitae of intestinal intraepithelial $\mathrm{T}$ cells: their developmental and behavioral characteristics. Immunol Rev. 2007;215:154-65.

4. Cheroutre H, Lambolez F, Mucida D. The light and dark sides of intestinal intraepithelial lymphocytes. Nat Rev Immunol. 2011;11:445-56.

5. van de Wal Y, Corazza N, Allez M, et al. Delineation of a CD1drestricted antigen presentation pathway associated with human and mouse intestinal epithelial cells. Gastroenterology. 2003;124:1420-31.

6. Sugimoto K, Ogawa A, Mizoguchi E, et al. IL-22 ameliorates intestinal inflammation in a mouse model of ulcerative colitis. J Clin Investig. 2008;118:534-44.

7. Khwaja A, Rodriguez-Viciana P, Wennström S, et al. Matrix adhesion and Ras transformation both activate a phosphoinositide 3-OH kinase and protein kinase B/Akt cellular survival pathway. EMBO J. 1997;16:2783-93.

8. Nozaki K, Mochizuki W, Matsumoto Y, et al. Co-culture with intestinal epithelial organoids allows efficient expansion and motility analysis of intraepithelial lymphocytes. J Gastroenterol. 2016;51:206-13.

9. Sato T, Vries RG, Snippert HJ, et al. Single Lgr5 stem cells build crypt-villus structures in vitro without a mesenchymal niche. Nature. 2009;459:262-5.

10. Cheroutre H. Starting at the beginning: new perspective on the biology of mucosal T cells. Annu Rev Immunol. 2004;22:217-46.

11. Sujino T, London M, Hoytema van Konijnenburg DP, et al. Tissue adaptation of regulatory and intraepithelial CD4+ T cells controls gut inflammation. Science. 2016;352:1581-6.

12. Kitamoto S, Nagao-Kitamoto H, Kuffa P, et al. Regulation of virulence: the rise and fall of gastrointestinal pathogens. J Gastroenterol. 2016;51:195-205.

13. Hsiao EY, McBride SW, Hsien S, et al. Microbiota modulate behavioral and physiological abnormalities associated with neurodevelopmental disorders. Cell. 2013;155:1451-63.

14. Nanno M, Kanari Y, Naito T, et al. Exacerbating role of gammadelta $\mathrm{T}$ cells in chronic colitis of T-cell receptor alpha mutant mice. Gastroenterology. 2008;134:481-90.

15. Kadowaki A, Miyake S, Saga R, et al. Gut environment-induced intraepithelial autoreactive $\mathrm{CD} 4(+) \mathrm{T}$ cells suppress central nervous system autoimmunity via LAG-3. Nat Commun. 2016;7:11639. 